\title{
BIMIFICATION: HOW TO CREATE BIM FOR RETROFITTING
}

\author{
Raimar J. Scherer ${ }^{1}$ and Peter Katranuschkov ${ }^{2}$
}

\begin{abstract}
We propose a new approach towards the creation of a building information model of an existing building for the purpose of retrofitting, based on the standard IFC specification (ISO 16739). It implies a process, which we define as BIMification. This process undergoes two major phases: (1) Anamnesis, dedicated to the survey and collection of facts about the building, and (2) Diagnosis, dedicated to the analysis and interpretation of the collected facts to obtain the necessary understanding of the building and its performance and prepare for the retrofitting design. The paper outlines the broader research goal that triggered the development of the suggested approach, presents its concept and supporting ICT platform, and draws conclusions with regard to the envisaged impact. The research work builds upon findings from the EU FP7 projects ISES and eeEmbedded while going distinctly beyond their objectives and scope.
\end{abstract}

Keywords: BIM, existing building stock, energy efficient performance, retrofitting

\section{INTRODUCTION}

BIM is rapidly advancing as the most efficient approach to cooperative building design and construction today. However, BIM methodology is still mainly applied for new building projects. On the other side, there is a strong need to improve the quality and the overall performance of the existing building stock, especially with regard to energy use. The Energy Performance of Buildings Directive of the EU (EPBD 2010; 2012) and its 2016 update specify the application of various requirements to existing buildings, building units and building elements that are subject to retrofitting or major renovation towards achievement of near zero energy housing at cost-optimal levels. Using BIM as basis is of utmost importance for a successful answer to this challenge. However, unlike new designed buildings where BIM is created from scratch with the help of a BIM/CAD system like Autodesk's Revit or Nemetschek's Allplan, for existing buildings it has to be created from the actual 'as-built' state with practically none or only scarce building documentation in the form of incomplete 2D drawings.

In this paper we propose a new approach towards the creation of the building information model of an existing building subject to renovation or retrofitting. We coin the approach as BIMification, which we define as the process to obtain from an existing real building or a virtual 3D model of that building a BIM model based on the ISO 16739 data schema, IFC. In the following chapters we describe the background and the broader research goal that led to the proposed BIMification approach and present the underlying concept, methodology and envisaged ICT platform to support it.

1 Professor, Head of the Institute of Construction Informatics, Faculty of Civil Engineering, TU Dresden, Germany, raimar.scherer@tu-dresden.de

2 Senior Researcher, Institute of Construction Informatics, Faculty of Civil Engineering, TU Dresden, Germany, peter.katranuschkov@tu-dresden.de 


\section{RESEARCH AIM}

The overall goal of our research is the development of a new integrated BIM design studio for the renovation of aged buildings that can combine holistically BIM-based design and performance analysis. It will build upon a new 3-stage design process of Anamnesis - Diagnosis - Therapy (ADT), adapting and redefining a recent approach proposed for the rehabilitation of cultural heritage (Van Balen and Verstrynge 2016) to enable deep understanding of the existing building, its local environment and its inhabitants, a comprehensive picture of the pros and cons of the investments for retrofitting and the benefits for the owner and the users already at the earliest stages of the design process. Highly important for the envisaged overall process, shown schematically on Figure 1, is the successful solution of the following two challenges:

- Development of BIM for existing buildings through post-built BIMification, thereby enabling grounded anamnesis and diagnosis

- Development of an overall steering methodology based on consistent use of BIM and governed by a Key Point system of key design parameters, key performance indicators and key decision values, thereby enabling optimal therapy, i.e. achievement of an optimal retrofitting concept.

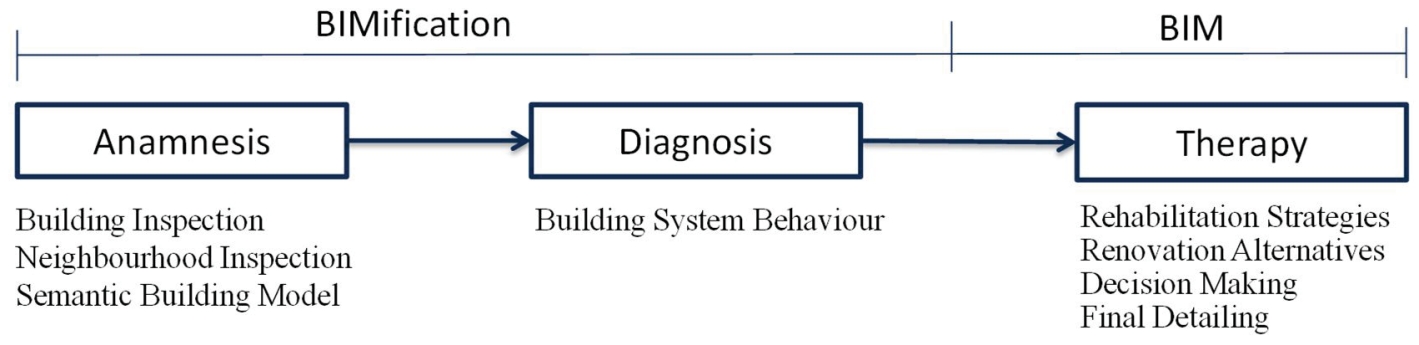

Figure 1: Proposed 3-stage method for retrofitting design

The proposed solution for the first of these two challenges is discussed in the paper. The second is currently researched in the eeEmbedded project regarding the energy efficient design of new buildings (Guruz and Scherer 2016). It is equally applicable for the renovation and retrofitting of the existing building stock.

The suggested overall approach is not depending on any specifically addressed time period but the knowledge templates which are an important part of the BIMification process have to conform to certain architectural styles. Therefore, as particular target, we have chosen the period 1960-1980. In this period, in order to reduce the ongoing housing shortage and to re-house people living in substandard homes, local authorities across Europe built a large number of residential tower blocks. However, public disapproval of these blocks quickly grew. Poor design, repetitive concrete exteriors and numerous technical deficiencies all contributed to dissatisfaction. Therefore, buildings of this period, having comparatively simple architectural layout, are a major target of retrofitting actions today and hence a logical first practical target of our research.

\section{Suggested BiMification Process}

An efficient renovation strategy needs a solid information base about the building and its past and future use, including the expected behaviour of the users in the planned new technological environment and their current energy-related behaviour. At present, BIM documentation does not exist nor is information about the building continuously updated 
or monitored. Therefore, BIMification is a prerequisite for efficient BIM-based retrofitting design. It is a major part of the first two stages of the ADT process, i.e.:

- Anamnesis, dedicated to the survey and collection of facts about the building

- Diagnosis, dedicated to the analysis and interpretation of the collected facts to obtain the necessary understanding of the building and its performance

Once the BIM is constructed, the final Therapy stage can be conducted, using advanced BIM-based analysis and simulation methods to predict the expected improved energy performance and the related lifecycle costs from the application of the proposed retrofitting measures and to evaluate different retrofitting proposals.

The Anamnesis phase includes five tasks, sub-structured in basic and advanced BIMification. The first comprises:

- Geometrical BIMification, which is the pure geometric model extracted from observation of the existing building,

- Topological BIMification, which provides a first simple topological model, rendering the adjacency of spaces and zones and their usage, and

- Neighbourhood BIMification, which is the relevant neighbourhood geometry model and the infrastructure information.

The second comprises:

- Element BIMification, which is the structuring of the geometric model in correctly assembled building elements expressed in IFC and modelling the as-built situation, for which former construction knowledge represented in the form of knowledge templates can be applied, thereby leading to the technical morphological model and the topological element model complementing the topological space model from above, and

- Behaviour BIMification, which is the semantic enrichment of the building elements, i.e. the morphological model with additional technical information about the element behaviour (like heat or moisture transfer, structural strength etc.), based on a system identification method and leading to IFC elements enriched with performance information.

For a sufficiently sound basis for the development of good renovation concepts, there are two more BIMification tasks needed. They define the Diagnosis phase, which we summarize as extended BIMification, namely:

- Gap BIMification, which should identify building elements and groups with critical or bad performance and can be accomplished using dedicated performance analyses, and

- Variability BIMification, which identifies the response of building elements and systems to changes; it can be accomplished by means of sensitivity analysis of the energy performance.

These tasks are discussed in more detail in the following section.

\subsection{Basic BIMification}

Today, data gathering from existing buildings, regarding their history, damages, moisture problems, energetic and inhabitant behaviour and the energy infrastructure of the district, is a tedious and very time consuming process because the data is not stored in BIM. It is often inconsistent, provided in proprietary formats and not integrated in a $3 \mathrm{D}$ 
model of the building. Gathered in such a way, it can hardly be verified in terms of reliability, accuracy and consistency. Therefore, the first task of the BIMification process is the acquisition of the information about the existing building using state of the art 3D capturing for 3D model generation and alignment of the set of indoor 3D models and the outdoor model to obtain a geometrical BIM model (Previtali et al. 2014). Data from the building and neighbourhood survey can thereby be collected and integrated with observed thermal and permeability data. Methods and tools for the achievement of that task have already been elaborated in recent years including primitive extraction (Oesau et al. 2014) and local modelling via a surface model approach (Sahin 2015). The derived geometry can then be converted to IFC proxy objects that can be further processed by a CAD system or an envisaged $n D$ Navigator. On that basis a complementary topological model that represents basic adjacency information can be derived. Neighbourhood BIMification is at this stage restricted to the envelope geometry of the surrounding buildings and the terrain properties that can be taken from available GIS data or geodetic survey.

\subsection{Advanced BIMification}

Structuring the geometrical model to appropriately identified building elements needs further information. This is often tackled by annotating the geometrical model with some element semantics, but being almost pure manual work it is not sufficient for the BIMification process. To establish a reliable BIM model that can be used in the further steps of the ADT process, semantic enrichment of the building elements based on a BIM ontology is necessary. Accordingly, Element BIMification comprises (1) Mapping the proxy objects to appropriate instances of IFC building element subclasses such as IfcWall, IfcWindow etc. and their further subclasses like IfcWallStandardCase, using class level ontology queries and rules, (2) Mapping the generated modelling instances to a reduced ontology representation (without detailed geometry) using an available IfcOWL tool (Beetz et al. 2008), (3) Checking the resultant ontology model for correctness via model checking rules, and (4) Applying appropriate templates for old constructions to fill in missing technical information. There is hardly research in construction IT for the envisaged mapping process but ontology work from the eeEmbedded project (Kadolsky 2016) provides a good development basis.

The following identification of behavioural information about the building and HVAC elements is a complex task requiring a large area of expertise. We suggest limiting this task to the definition of an energy domain model of relatively low level of detail and applying an impulse response method, where the energy systems are switched on certain levels of power for a certain time providing an impulse like energy impact on the building's thermal behaviour. To monitor that behaviour, room temperatures can be measured via mobile sensors. Using appropriate simulation software such as EnergyPlus the thermal performance based on the acquired model data can be computed, compared with the measured data and adjusted to the actual behaviour by a sensitivity-based approach adapted from the ISES project (Katranuschkov et al. 2016). Its essence is to vary selected model parameters in structured manner until certain convergence with the measured performance is achieved. This method is known as simulation-based system identification. It requires high computing power that can be provided by a compute cloud and a method for fast generation of multiple model variations (i.e. thermal parameters and their combinations). It is also known as a greybox model approach, because it combines a simplified physical system with a data mining approach. 


\subsection{Extended BIMification}

Two more BIMification tasks are required to complete the diagnostic stage. The purpose of the first is Gap Analysis, i.e. the diagnosis of the weaknesses in the construction or the technical systems that should in first place draw the designer's attention or that may be resistant to renovation. Problems that have to be examined include structural weakness (e.g. needs of strengthening due to removal of load-bearing walls), moisture problems due to material deterioration, leaks in the technical systems, window insulation and corrosion. This can be done using performance analysis software with an especially developed supporting workflow to guide the user in the process and automate the needed tool interactions. Development of dedicated ontology rules as well as the use of knowledge templates for old buildings can help in finding the most likely targets.

The goal of Variability Analysis is to find the sensitivity of building elements and technical equipment to changes in order to determine as early as possible promising renovation targets or reject inefficient measures with regard to energy performance, user comfort and life cycle costs. To fulfil that goal, the created BIM model of the old building and the facts obtained in the anamnesis process and the gap analysis have to be combined with dedicated selective use of modern energy technologies, building elements and materials. This enables massive use of "what-if" scenarios at the very outset of the therapy process, aiming to find the response of the building to various changes. It can be accomplished by using sensitivity analysis, including both energy simulation and LCA/LCC assessment tools. Knowledge templates representing new elements and materials can be applied to replace existing model data from the old building without changing the basic BIM model used as "blueprint". The generated variants of the model can be simulated using cloud computing to parallelise the dozens or even hundreds of simulation runs that will be required to obtain reliable sensitivity results.

\subsection{Use of Knowledge Templates in the BIMification Process}

Behaviour, gap and variability BIMification as well as the later BIM-based generation of retrofitting design alternatives presume the use of knowledge templates to (1) substitute lack of information, (2) avoid manual detailing, and (3) enable easy generation of parameter variations and related simulation scenarios. Knowledge templates represent characteristic intelligent (sub)models for building elements and system components like façades, thermal insulation, energy buffers, energy control components, construction methods (old and modern) and may even represent partial systems like user activity or maintenance models. They can be classified in architectural detailing templates, engineering system templates, occupancy templates, climate templates, energy environment templates, district energy templates and facility management templates. Such templates can be provided in a generalized ontology-based knowledge framework and linked with the BIM/IFC objects following the approach suggested by Gudnason et al. (2014). Hereby, there are three subcategories that need to be applied:

- Knowledge templates for old construction elements and methods regarding (1) the bearing structure, enabling to decide to what extent the structuring system can be changed, and (2) the building insulations, including the roof structure (use of brick and concrete tiles, prefabricated roof trusses etc. and associated problems like rain penetration, infiltration or cooling), the walls (brick, concrete and cavity walls and associated typical moisture problems), the windows (timber casing, glazing, air tightness etc.), the ground floors and the foundations where the main deficiencies with regard to energy performance can be found 
- Knowledge templates for old energy systems regarding (1) the used typical HVAC systems, (2) the typical energy supply (local or district heating, electrification, water resources and the associated typical $\mathrm{CO}_{2}$ footprints), and (3) typical performance problems (for the more efficient recognition of gaps)

- Knowledge templates for modern renovation BIM and BEM design that will help to reduce the complexity of the design by enabling quick integration of all replacement elements and subsystems in the generated BIM model of the old building (moreover, by varying template parameters or exchanging a template by another, retrofitting concepts can be quickly varied and verified).

\subsection{Generation of Simulation Alternatives}

Automated generation of simulation alternatives is another important aspect of the suggested BIMification process. Without an adequate approach, the envisaged multiple simulations for system identification and for the evaluation of design alternatives cannot be practically achieved. Fortunately, definition of design alternatives and sensitivity analysis studies can be efficiently represented by means of a Simulation Matrix, without the need to duplicate the huge amount of BIM data where only a few design parameters have to be varied (Katranuschkov et al. 2016).

The suggested simulation matrix allows the representation of more than one building model as well as orientation, climate, occupancy, extended material properties and other external resources in a single XML schema, consistently interlinking the information resources needed for the energy simulations. Furthermore, variant building settings can be stored and exchanged between the design partners during several project phases. Each specific matrix instance comprises four sections: (1) Variables, describing the varying properties of the building at the level of location, zones, element and material, (2) Targets, defining the required simulation scope (single space, zone, storey, whole building), (3) Assignment groups, providing the links between the specified variable properties and the actual BIM objects (spaces, elements, equipment) to which they are related, and (4) Combinations, interlinking Targets, Variables and Assignment groups to define the separate simulations to be prepared and executed in the cloud environment.

\section{FUNCTIONAL ICT CONCEPT}

The essence of the functional ICT concept for BIM-based retrofitting support, incorporating the described BIMification tasks, is to separate the whole ADT process in five interacting cycles as shown schematically in Figure 2 below. Three of these cycles can be highly automated to release the designer from time-consuming manual data, information and knowledge management tasks. The model management cycle is the steering cycle via which the four other cycles and their related ICT components are interacting, and the BIMification cycle is the prerequisite making the BIM-based approach for the retrofitting of existing buildings possible. The individual retrofitting tasks are thereby not seen as separate tasks for which separate ICT environments need to be provided, but as one coherent common platform. With this approach, a single optimized task and context aware working platform can be established.

According to this functional concept the technical software architecture is organized in 5 blocks: (1) BIM kernel component comprising the BIM middleware services (incl. multi-model combining, filtering, mapping, configuring), the model repositories, the simulation management services, the process and collaboration management services 
and the cloud access interface, (2) BIMification component where the tools for the anamnesis and diagnosis tasks are located, (3) Numerical component comprising the various multi physics analysis tools as well as LCA, LCC and ROI simulation tools, (4) Knowledge component containing the strategies based on stored best practice examples and the knowledge templates for fast principal detailing and building element identification, and (5) BIM design component where the main modules are BIM modelling (CAD), inspection (nD Navigator) and a key point design guideline system.

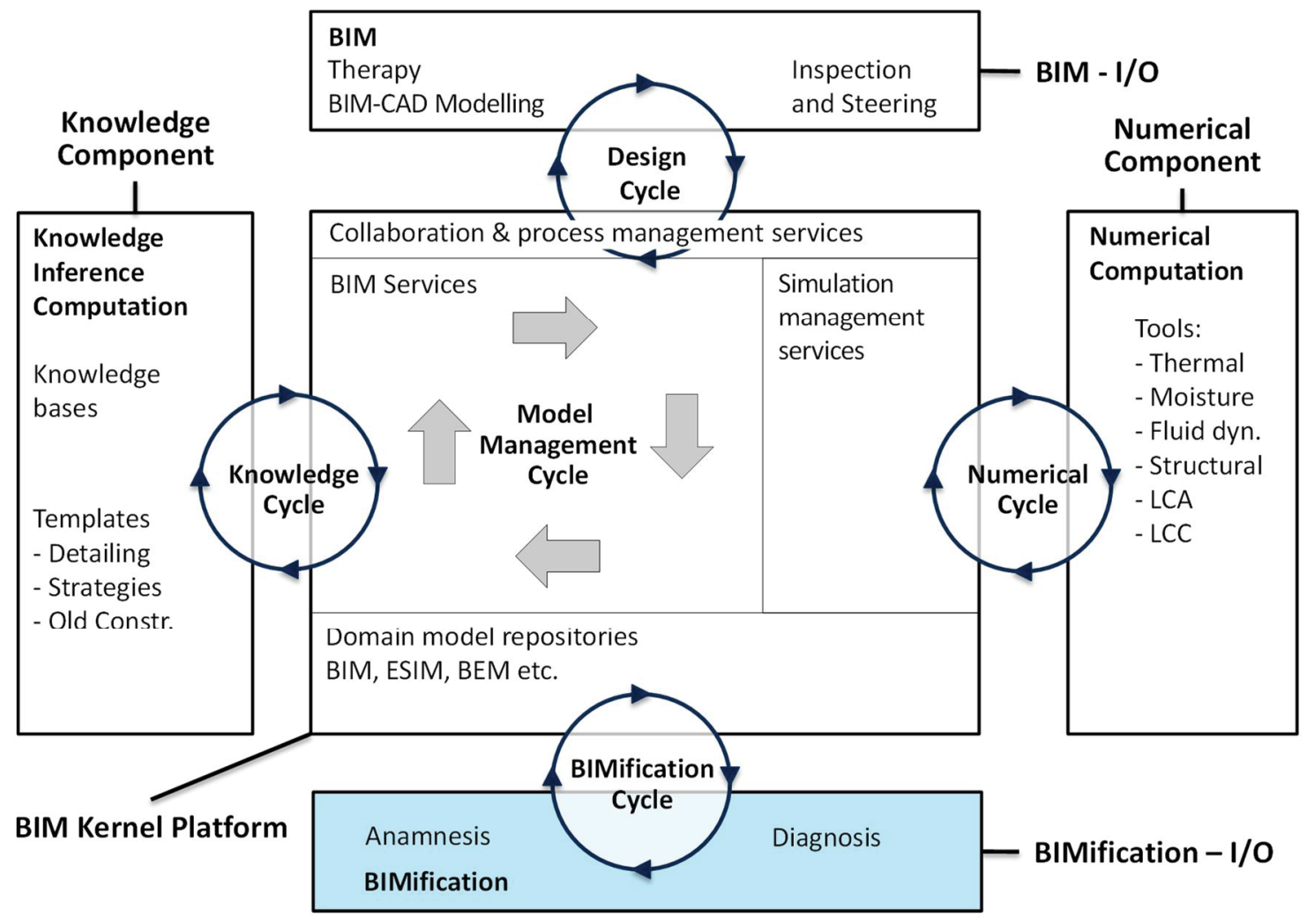

Figure 2: Suggested high-level functional architecture for efficient BIM-based retrofitting design support

\section{CONCLUSIONS}

The proposed approach for the creation and use of BIM in the retrofitting and renovation of old buildings presents a new methodology that is yet in an early development phase. However, while there are no results with regard to the full process yet, several methods supporting the realization of the described BIMification steps have already been successfully developed within the ISES and eeEmbedded projects. Such methods include the semi-automated identification of the building façade, various BIM querying and filtering methods, the definition and use of knowledge templates to compensate the lack of actual data, rule-based reasoning with regard to technical systems and building elements and so on.

The suggested BIMification process will be tested on real buildings from the period 1960-1980 where massive construction of affordable dwellings has taken place across Europe and hence strong need for renovation and retrofitting exists. By enabling sound and well-structured BIM creation, it is expected to bring significant benefits with regard to behaviour building system identification, reliable planning of retrofitting measures 
both in terms of energy performance and costs and, last but not least, measurable contributions with regard to the creation of building models of existing buildings.

\section{ACKNOWLEDGMENTS}

The research presented in this paper has been made possible with the financial support of the TU Dresden and the European Commission, in the frames of the projects ISES (Grant no. 288819, 2012-16) and eeEmbedded (Grant no. 609349, 2013-17), as well as the contribution of various project partners. This support is gratefully acknowledged.

\section{REFERENCES}

Beetz, J., van Leeuwen, J., and de Vries, B. (2008). IfcOWL: A Case of Transforming EXPRESS Schemas into Ontologies. AI EDAM, Vol. 23, Issue 1 (Developing and Using Engineering Ontologies), pp. 89-101.

EPBD (2010-12). Directive 2010/31/EU of the European Parliament and of the Council, Pub. L. No. 2010/31/EU, Recast: Directive 2012/27/EU of the European Parliament and of the Council, Pub. L. No. 2012/27/EU.

Gudnason, G., Katranuschkov, P., Balaras, C., and Scherer, R. J. (2014). Framework for Interoperability of Information Resources in the Building Energy Simulation Domain. Proc. ICCCBE 2014 and CIB W78 Conference, Orlando, USA.

Guruz, R., and Scherer, R. J. (2014). Sustainable Energy Entrepreneurship through Architectural Design: A Key Point Controlled Method. Entrepreneurship and Sustainability Issues 2/2 (2014), ISSN 2345-0282.

ISO 16739 (2013). Industry Foundation Classes (IFC) for data sharing in the construction and facility management industries. (C) ISO, Geneva.

Kadolsky, M. (2016). eeEmbedded Interoperability and Ontology Framework. eeEmbedded Deliverable D5.1, ( ) eeEmbedded Consortium, Brussels, 52 p.

Katranuschkov, P., Scherer, R. J., and Hoch, R. (2016). Optimizing Energy Efficient Building Design Using BIM. Proc. ICCCBE 2016 Conference, Osaka, Japan, Paper ID 113.

Oesau, S., Lafarge, F., and Alliez, P. (2014). Indoor Scene Reconstruction using Feature Sensitive Primitive Extraction and Graph-cut, ISPRS Journal of Photogrammetry and Remote Sensing, Elsevier, 2014/90, pp. 68-82.

Previtali, M., Barazzetti, L., Brumana, R., and Scaioni, M. (2014). Towards Automatic Indoor Reconstruction of Cluttered Building Rooms from Point Clouds. ISPRS Annals of the Photogrammetry, Remote Sensing and Spatial Information Sciences, Vol. II-5.

Sahin, C. (2015). Planar Segmentation of Indoor Terrestrial Laser Scanning Point Clouds via Distance Function from a Point to a Plane, Optics and Lasers in Engineering 01/2015, 64:23-31, DOI: 10.1016/j.optlaseng.2014.07.007.

Van Balen, K, and Verstrynge, E. (2016). Structural Analysis of Historical Constructions: Anamnesis, Diagnosis, Therapy, Controls. Proc. 10th Int. Conference. on Structural Analysis of Historical Constructions, Leuven, Belgium, 13-15 Sep. 2016, CRC Press, ISBN 978-1-138-02951-4, $312 \mathrm{p}$. 